\title{
ON SEMI-MODULAR SUBALGEBRAS OF LIE ALGEBRAS OVER FIELDS OF ARBITRARY CHARACTERISTIC
}

\author{
DAVID A. TOWERS \\ Department of Mathematics, Lancaster University \\ Lancaster LA1 4YF, England \\ email: d.towers@lancaster.ac.uk
}

\begin{abstract}
This paper is a further contribution to the extensive study by a number of authors of the subalgebra lattice of a Lie algebra. It is shown that, in certain circumstances, including for all solvable algebras, for all Lie algebras over algebraically closed fields of characteristic $p>0$ that have absolute toral rank $\leq 1$ or are restricted, and for all Lie algebras having the one-and-a-half generation property, the conditions of modularity and semi-modularity are equivalent, but that the same is not true for all Lie algebras over a perfect field of characteristic three. Semi-modular subalgebras of dimensions one and two are characterised over (perfect, in the case of two-dimensional subalgebras) fields of characteristic different from 2,3 .
\end{abstract}

Keywords: Lie algebra; subalgebra lattice; modular; semi-modular, quasi-ideal.

AMS Subject Classification: 17B05, 17B50, 17B30, 17B20

\section{Introduction}

This paper is a further contribution to the extensive study by a number of authors of the subalgebra lattice of a Lie algebra, and is, in part, inspired by the papers of Varea $([15],[16])$. A subalgebra $U$ of a Lie algebra $L$ is called

- modular in $L$ if it is a modular element in the lattice of subalgebras of $L$; that is, if

$$
<U, B>\cap C=<B, U \cap C>\quad \text { for all subalgebras } B \subseteq C,
$$

and

$$
<U, B>\cap C=\langle B \cap C, U>\quad \text { for all subalgebras } U \subseteq C,
$$

(where, $<U, B>$ denotes the subalgebra of $L$ generated by $U$ and $B$ ); 
- upper modular in $L$ (um in $L$ ) if, whenever $B$ is a subalgebra of $L$ which covers $U \cap B$ (that is, such that $U \cap B$ is a maximal subalgebra of $B$ ), then $<U, B>$ covers $U$;

- lower modular in $L(\operatorname{lm}$ in $L)$ if, whenever $B$ is a subalgebra of $L$ such that $<U, B>$ covers $U$, then $B$ covers $U \cap B$;

- semi-modular in $L$ (sm in $L$ ) if it is both u.m. and l.m. in $L$.

In this paper we extend the study of sm subalgebras started in [12]. In section two we give an example of a Lie algebra over a perfect field of characteristic three which has a sm subalgebra that is not modular. However, it is shown that for all solvable Lie algebras, and for all Lie algebras over an algebraically closed field of characteristic $p>0$ that have absolute toral rank $\leq 1$ or are restricted, the conditions of modularity, semi-modularity and being a quasi-ideal are equivalent. The latter extends results of Varea in [16] where the characteristic of the field is restricted to $p>7$. It is then shown that for all Lie algebras having the one-and-a-half generation property the conditions of modularity and semi-modularity are equivalent.

In section three, sm subalgebras of dimension one are studied. These are characterised over fields of characteristic different from 2,3. This result generalises a result of Varea in [15] concerning modular atoms. In the fourth section we show that, over a perfect field of characteristic different from 2,3, the only Lie algebra containing a two-dimensional core-free sm subalgebra is $s l_{2}(F)$. It is also shown that, over certain fields, every sm subalgebra that is solvable, or that is split and contains the normaliser of each of its non-zero subalgebras, is modular.

Throughout, $L$ will denote a finite-dimensional Lie algebra over a field $F$. There will be no assumptions on $F$ other than those specified in individual results. The symbol ' $\oplus$ ' will denote a vector space direct sum. If $U$ is a subalgebra of $L$, the core of $U, U_{L}$, is the largest ideal of $L$ contained in $U$; we say that $U$ is core-free if $U_{L}=0$. We denote by $R(L)$ the solvable radical of $L$, by $Z(L)$ the centre of $L$, and put $C_{L}(U)=\{x \in L:[x, U]=0\}$.

\section{General results}

We shall need the following result from [12].

Lemma 2.1 Let $U$ be a proper sm subalgebra of a Lie algebra $L$ over an arbitrary field $F$. Then $U$ is maximal and modular in $\langle U, x\rangle$ for all $x \in L \backslash U$. 
Proof: We have that $U$ is maximal in $\langle U, x\rangle$, by Lemma 1.4 of [12], and hence that $U$ is modular in $\langle U, x\rangle$, by Theorem 2.3 of [12]

In [12] it was shown that, over fields of characteristic zero, $U$ is modular in $L$ if and only if it is sm in $L$. This result does not extend to all fields of characteristic three, as we show next. Recall that a simple Lie algebra is split if it has a splitting Cartan subalgebra $H$; that is, if the characteristic roots of $\operatorname{ad}_{L} h$ are in $F$ for every $h \in H$. Otherwise we say that it is non-split.

Proposition 2.2 Let $L$ be a Lie algebra of dimension greater than three over an arbitrary field $F$, and suppose that every two linearly independent elements of $L$ generate a three-dimensional non-split simple Lie algebra. Then there are maximal subalgebras $M_{1}, M_{2}$ of $L$ such that $M_{1} \cap M_{2}=0$.

Proof: This is proved in Proposition 4 of [8].

\section{Example}

Let $G$ be the algebra constructed by Gein in Example 2 of [7]. This is a seven-dimensional Lie algebra over a certain perfect field $F$ of characteristic three. In $G$ every linearly independent pair of elements generate a threedimensional non-split simple Lie algebra. It follows from Proposition 2.2 above that there are two maximal subalgebras $M, N$ in $G$ such that $M \cap N=$ 0 . Choose any $0 \neq a \in M$. Then $\langle a, N\rangle \cap M=M$, but $\langle N \cap M, a\rangle=$ $F a$, so $F a$ is not a modular subalgebra of $L$. However, it is easy to see that all atoms of $G$ are sm in $G$.

A subalgebra $Q$ of $L$ is called a quasi-ideal of $L$ if $[Q, V] \subseteq Q+V$ for every subspace $V$ of $L$. It is easy to see that quasi-ideals of $L$ are always semimodular subalgebras of $L$. When $L$ is solvable the semi-modular subalgebras of $L$ are precisely the quasi-ideals of $L$, as the next result, which is based on Theorem 1.1 of [15], shows.

Theorem 2.3 Let $L$ be a solvable Lie algebra over an arbitrary field $F$ and let $U$ be a proper subalgebra of $L$. Then the following are equivalent:

(i) $U$ is modular in $L$;

(ii) $U$ is sm in $L$; and

(iii) $U$ is a quasi-ideal of $L$. 
Proof: (i) $\Rightarrow$ (ii) : This is straightforward.

(ii) $\Rightarrow$ (iii) : Let $L$ be a solvable Lie algebra of smallest dimension containing a subalgebra $U$ which is sm in $L$ but is not a quasi-ideal of $L$. Then $U$ is maximal and modular in $L$, by Lemma 2.1, and $U_{L}=0$. Let $A$ be a minimal ideal of $L$. Then $L=U+A$. Moreover, $U \cap A$ is an ideal of $L$, since $A$ is abelian, whence $U \cap A=0$ and $L=U \oplus A$. Now $U$ is covered by $\langle U, A>$ so $A$ covers $U \cap A=0$. This yields that $\operatorname{dim} A=1$ and so $U$ is a quasi-ideal of $L$, a contradiction.

(iii) $\Rightarrow$ (i) : This is straightforward.

Corollary 2.4 Let $L$ be a solvable Lie algebra over an arbitrary field $F$ and let $U$ be a core-free sm subalgebra of $L$. Then $\operatorname{dim}(U)=1$ and $L$ is almost abelian.

Proof: This follows from Theorem 2.3 and Theorem 3.6 of [1].

We now consider the case when $L$ is not necessarily solvable. First we shall need the following result concerning $\mathrm{psl}_{3}(F)$.

Proposition 2.5 Let $F$ be a field of characteristic 3 and let $L=p l_{3}(F)$. Then $L$ has no maximal sm subalgebra.

Proof: Let $E_{i j}$ be the $3 \times 3$ matrix that has 1 in the $(i, j)$-position and 0 elsewhere, and denote by $\overline{E_{i j}}$ the canonical image of $E_{i j} \in \operatorname{sl}_{3}(F)$ in $\operatorname{psl}_{3}(F)$. Put $e_{-3}=\overline{E_{23}}, e_{-2}=\overline{E_{31}}, e_{-1}=\overline{E_{12}}, e_{0}=\overline{E_{11}}-\overline{E_{22}}, e_{1}=\overline{E_{21}}, e_{2}=\overline{E_{13}}$, $e_{3}=\overline{E_{32}}$. Then $e_{-3}, e_{-2}, e_{-1}, e_{0}, e_{1}, e_{2}, e_{3}$ is a basis for $p l_{3}(F)$ with

$\left[e_{0}, e_{i}\right]=e_{i}$ if $i>0,\left[e_{0}, e_{i}\right]=-e_{i}$ if $i<0, \quad\left[e_{-i}, e_{j}\right]=\delta_{i j} e_{0}$ if $i, j>0$ and $\left[e_{i}, e_{j}\right]=e_{-k}$ for every cyclic permutation $(i, j, k)$ of $(1,2,3)$ or $(-3,-2,-1)$.

Put $B_{i, j}=F e_{0}+F e_{i}+F e_{j}$ for each non-zero $i, j$. If $i, j$ are of opposite sign then $B_{i, j}$ is a subalgebra, every maximal subalgebra of which is two dimensional.

Let $M$ be a maximal sm subalgebra of $L$. For each $i, j$ of opposite sign, if $B_{i, j} \nsubseteq M$ then $M \cap B_{i, j}$ is two dimensional. Since $M$ is at most fivedimensional, by considering the intersection with each of $B_{1,-1}, B_{2,-2}$ and $B_{3,-3}$ it is easy to see that $e_{0} \in M$. But then, considering $B_{1,-1}$ again, we have either $e_{1} \in M$ or $e_{-1} \in M$. Suppose the former holds. Taking the intersection of $M$ with $B_{2,-3}$ shows that $e_{-3} \in M$; then with $B_{2,-1}$ gives $e_{2} \in M$; next with $B_{3,-2}$ gives $e_{-2} \in M$; finally with $B_{3,-1}$ yields $e_{3} \in M$. 
But then $M=L$, a contradiction. A similar contradiction is easily obtained if we assume that $e_{-1} \in M$.

Let $\left(L_{p},[p], \iota\right)$ be any finite-dimensional $p$-envelope of $L$. If $S$ is a subalgebra of $L$ we denote by $S_{p}$ the restricted subalgebra of $L_{p}$ generated by $\iota(S)$. Then the (absolute) toral rank of $S$ in $L, T R(S, L)$, is defined by

$$
T R(S, L)=\max \left\{\operatorname{dim}(T): T \text { is a torus of }\left(S_{p}+Z\left(L_{p}\right)\right) / Z\left(L_{p}\right)\right\} .
$$

This definition is independent of the $p$-envelope chosen (see [11]). We write $T R(L, L)=T R(L)$. Then, following the same line of proof, we have an extension of Lemma 2.1 of [16].

Lemma 2.6 Let L be a Lie algebra over an algebraically closed field of characteristic $p>0$ such that $T R(L) \leq 1$. Then the following are equivalent:

(i) $U$ is modular in $L$;

(ii) $U$ is sm in $L$; and

(iii) $U$ is a quasi-ideal of $L$.

Proof: We need only show that (ii) $\Rightarrow$ (iii). Let $U$ be a sm subalgebra of $L$ that is not a quasi-ideal of $L$. Then there is an $x \in L$ such that $<U, x>\neq U+F x$. We have that $U$ is maximal and modular in $\langle U, x\rangle$, by Lemma 2.1, and $\langle U, x\rangle$ is not solvable, by Theorem 2.3. Furthermore $T R(<U, x>) \leq T R(L) \leq 1$, by Proposition 2.2 of [11], and $\langle U, x\rangle$ is not nilpotent so $T R(\langle U, x\rangle) \neq 0$, by Theorem 4.1 of [11], which yields $T R(<U, x>)=1$. We may therefore suppose that $U$ is maximal and modular in $L$, of codimension greater than one in $L$, and that $T R(L)=1$.

Put $L^{\infty}=\bigcap_{n>1} L^{n}$. Suppose first that $R\left(L^{\infty}\right) \not \leq U$. Then $U \cap R\left(L^{\infty}\right)$ is maximal and modular in the solvable subalgebra $R\left(L^{\infty}\right)$, so $U \cap R\left(L^{\infty}\right)$ has codimension one in $R\left(L^{\infty}\right)$. Since $U$ is maximal in $L$ we have $L=U+R\left(L^{\infty}\right)$ and so $\operatorname{dim}(L / U)=1$, which is a contradiction. This yields that $R\left(L^{\infty}\right) \leq$ $U$. Moreover, $L^{\infty} \not \leq U$, since this would imply that $U / L^{\infty}$ is maximal in the nilpotent algebra $L / L^{\infty}$, giving $\operatorname{dim}(L / U)=1$, a contradiction again. It follows that $\left(U \cap L^{\infty}\right) / R\left(L^{\infty}\right)$ is modular and maximal in $L^{\infty} / R\left(L^{\infty}\right)$. But now $L^{\infty} / R\left(L^{\infty}\right)$ is simple, by Theorem 2.3 of [17], and $1=T R(L) \geq$ $T R\left(L^{\infty}, L\right) \geq T R\left(L^{\infty} / R\left(L^{\infty}\right)\right)$ by section 2 of [11], so $T R\left(L^{\infty} / R\left(L^{\infty}\right)\right)=1$. This implies that

$$
p \neq 2, \quad L^{\infty} / R\left(L^{\infty}\right) \in\left\{s l_{2}(F), W(1: \underline{1}), H(2: \underline{1})^{(1)}\right\} \text { if } p>3
$$




$$
\text { and } \quad L^{\infty} / R\left(L^{\infty}\right) \in\left\{s l_{2}(F), p s l_{3}(F)\right\} \text { if } p=3,
$$

by [9] and [10].

Now $H(2: \underline{1})^{(1)}$ has no modular and maximal subalgebras, by Corollary 3.5 of [15]; likewise $\operatorname{psl}_{3}(F)$ by Proposition 2.5. It follows that $L^{\infty} / R\left(L^{\infty}\right)$ is isomorphic to $W(1: \underline{1})$, which has just one proper modular subalgebra and this has codimension one, by Proposition 2.3 of [15], or to $s l_{2}(F)$ in which the proper modular subalgebras clearly have codimension one. Hence $\operatorname{dim}\left(L^{\infty} /\left(U \cap L^{\infty}\right)=1\right.$. Since $L=U+L^{\infty}$ we conclude that $\operatorname{dim}(L / U)=$ $\operatorname{dim}\left(L^{\infty} /\left(U \cap L^{\infty}\right)=1\right.$. This contradiction gives the claimed result.

We then have the following extension of Theorem 2.2 of [16]. The proof is virtually as given in [16], but as the restriction to characteristic $>7$ has been removed the details need to be checked carefully. The proof is therefore included for the convenience of the reader.

Theorem 2.7 Let $L$ be a restricted Lie algebra over an algebraically closed field $F$ of characteristic $p>0$, and let $U$ be a proper subalgebra of $L$. Then the following are equivalent:

(i) $U$ is modular in $L$;

(ii) $U$ is sm in $L$; and

(iii) $U$ is a quasi-ideal of $L$.

Proof: As before it suffices show that (ii) $\Rightarrow$ (iii). Let $U$ be a sm subalgebra of $L$ that is not a quasi-ideal of $L$. Then there is an $x \in L$ such that $<U, x\rangle \neq U+F x$. First note that $\langle U, x\rangle$ is a restricted subalgebra of $L$. For, suppose not and pick $z \in<U, x>_{p}$ such that $z \notin<U, x>$. Since $<U, x>$ is an ideal of $\langle U, x\rangle_{p}$ we have that $\left.[z, U] \leq\langle U, x\rangle \cap<U, z\right\rangle$. But $U$ is maximal in $\langle U, z\rangle$, by Lemma 2.1, and so $\langle U, x\rangle \cap\langle U, z\rangle=$ $U$, giving $[z, U] \leq U$. But $U$ is self-idealizing, by Lemma 1.5 of [12], so $z \in U$. This contradiction proves the claim. So we may as well assume that $L=<U, x>$. Moreover, $U$ is restricted since it is self-idealizing, whence $\left(U_{L}\right)_{p} \leq U$. As $\left(U_{L}\right)_{p}$ is an ideal of $L$ we have that $U_{L}=\left(U_{L}\right)_{p}$. It follows that $L / U_{L}$ is also restricted. We may therefore assume that $U$ is a core-free modular and maximal subalgebra of $L$ of codimension greater than one in $L$.

Now $L$ is spanned by the centralizers of tori of maximal dimension, by Corollary 3.11 of [17], so there is such a torus $T$ with $C_{L}(T) \not \leq U$. Let $L=C_{L}(T) \oplus \sum L_{\alpha}(T)$ be the decomposition of $L$ into eigenspaces with 
respect to $T$. We have that $C_{L}(T)$ is a Cartan subalgebra of $L$, by Theorem 2.14 of [17]. It follows from the nilpotency of $C_{L}(T)$ and the modularity of $U$ that $U \cap C_{L}(T)$ has codimension one in $C_{L}(T)$.

Now let $L^{(\alpha)}=\sum_{i \in P} L_{i \alpha}(T)$, where $P$ is the prime field of $F$, be the 1 -section of $L$ corresponding to a non-zero root $\alpha$. From the modularity of $U$ we see that $U \cap L^{(\alpha)}$ is a modular and maximal subalgebra of $L^{(\alpha)}$. Since $U$ is core-free and self-idealizing, $Z(L)=0$. But then $T R(T, L)=$ $T R(L)$, since $T$ is a maximal torus, whence $T R\left(L^{(\alpha)}\right) \leq 1$, by Theorem 2.6 of [11]. It follows from Lemma 2.6 that $M \cap L^{(\alpha)}$ is a quasi-ideal of $L^{(\alpha)}$. As $U \cap L^{(\alpha)}$ is maximal in $L^{(\alpha)}$, we have that $\operatorname{dim}\left(L^{(\alpha)} /\left(U \cap L^{(\alpha)}\right)\right) \leq 1$ and $L^{(\alpha)}=U \cap L^{(\alpha)}+C_{L}(T)$. This yields that $L=U+C_{L}(T)$ and hence that $\operatorname{dim}(L / U)=\operatorname{dim}\left(C_{L}(T) /\left(U \cap C_{L}(T)\right)\right)=1$, a contradiction. The result follows.

We shall say that the Lie algebra $L$ has the one-and-a-half generation property if, given any $0 \neq x \in L$, there is an element $y \in L$ such that $<x, y\rangle=L$. Then we have the following result.

Theorem 2.8 Let $L$ be a Lie algebra, over any field $F$, which has the oneand-a-half generation property. Then every sm subalgebra of $L$ is a modular maximal subalgebra of $L$.

Proof: Let $U$ be a sm subalgebra of $L$ and let $0 \neq u \in U$. Then there is an element $x \in L$ such that $L=<u, x>=<U, x>$. It follows from Lemma 2.1 that $U$ is modular in $L$.

Corollary 2.9 Let $L$ be a Lie algebra over an infinite field $F$ of characteristic different from 2, 3 which is a form of a classical simple Lie algebra. Then every sm subalgebra of $L$ is a modular maximal subalgebra of $L$.

Proof: Under the given hypotheses $L$ has the one-and-a-half generation property, by Theorem 2.2.3 and section 1.2.2 of [3], or by [5].

We also have the following analogue of a result of Varea from [15].

Corollary 2.10 Let $F$ be an infinite perfect field of characteristic $p>2$, and assume that $p^{n} \neq 3$. Then the subalgebra $W(1: \mathbf{n})_{\mathbf{0}}$ is the unique sm subalgebra of $W(1: \mathbf{n})$.

Proof: Let $L=W(1: \mathbf{n})$ and let $\Omega$ be the algebraic closure of $F$. Then $L \otimes_{F} \Omega$ is simple and has the one-and-a-half generation property, by Theorem 
4.4.8 of [3]. It follows that $L$ has the one-and-a-half generation property (see section 1.2.2 of [3]). Let $U$ be a sm subalgebra of $L$. Then $U$ is modular and maximal in $L$ by Theorem 2.8. Suppose that $U \neq L_{0}$. Then $L=U+L_{0}$ and $U \cap L_{0}$ is maximal in $L_{0}$. But $L_{0}$ is supersolvable (see Lemma 2.1 of [13] for instance) so $\operatorname{dim}\left(L_{0} /\left(L_{0} \cap U\right)\right)=1$. It follows that $\operatorname{dim}(L / U)=$ $\operatorname{dim}\left(L_{0} /\left(L_{0} \cap U\right)\right)=1$, whence $U=L_{0}$, which is a contradiction.

\section{Semi-modular atoms}

We say that $L$ is almost abelian if $L=L^{2} \oplus F x$ with ad $x$ acting as the identity map on the abelian ideal $L^{2}$. A $\mu$-algebra is a non-solvable Lie algebra in which every proper subalgebra is one dimensional. A subalgebra $U$ of a Lie algebra $L$ is a strong ideal (respectively, strong quasi-ideal) of $L$ if every one-dimensional subalgebra of $U$ is an ideal (respectively, quasi-ideal) of $L$; it is modular* in $L$ if it satisfies a dualised version of the modularity conditions, namely

$$
<U, B>\cap C=<B, U \cap C>\quad \text { for all subalgebras } B \subseteq C,
$$

and

$$
<U \cap B, C>=<B, C>\cap U \quad \text { for all subalgebras } C \subseteq U \text {. }
$$

\section{Example}

Let $K$ be the three-dimensional Lie algebra with basis $a, b, c$ and multiplication $[a, b]=c,[b, c]=b,[a, c]=a$ over a field of characteristic two. Then $K$ has a unique one-dimensional quasi-ideal, namely $F c$. Thus for each $0 \neq u \in F c$ and $k \in K \backslash F c$ we have that $\langle u, k\rangle$ is two dimensional. However $K$ is not almost abelian. In fact $K$ is simple, $F c$ is core-free and is the Frattini subalgebra of $K$, and so any two linearly independent elements not in $F c$ generate $K$.

We shall need a result from [4]. However, because of the above example, there is a (slight) error in three results in this paper. The error comes from an incorrect use of Theorem 3.6 of [1]. The three corrected results are as follows:

Lemma 3.1 (Lemma 2.2 of [4]) If $Q$ is a strong quasi-ideal of $L$, then $Q$ is a strong ideal of $L$, or $L$ is almost abelian, or $F$ has characteristic two, $L=K$ and $Q=F c$. 
Proof: Assume that $Q$ is a strong quasi-ideal and that there exists $q \in Q$ such that $F q$ is not an ideal of $L$. Then Theorem 3.6 of [1] gives that $L$ is almost abelian, or $F$ has characteristic two, $L=K$ and $Q=F c$. The result follows.

The proof of the following result is the same as the original.

Proposition 3.2 (Proposition 2.3 of [4]) Let $Q$ be a proper quasi-ideal of a Lie algebra $L$ which is modular* in $L$. Then $Q$ is a strong quasi-ideal and so is given by Lemma 3.1.

Lemma 3.3 (Lemma 4.1 of [4]) Let $L$ be a Lie algebra over an arbitrary field $F$. Let $U$ be a core-free subalgebra of $L$ such that $\langle u, z\rangle$ is either two dimensional or a $\mu$-algebra for every $0 \neq u \in U$ and $z \in L \backslash U$. Then one of the following holds:

(i) L is almost abelian;

(ii) $\langle u, z>$ is a $\mu$-algebra for every $0 \neq u \in U$; and $z \in L \backslash U$

(iii) $F$ has characteristic two, $L=K$ and $F u=F c$.

Proof: This is the same as the original proof except that the following should be inserted at the end of sentence six: "or $\operatorname{char} F=2$ and $L=K$ ".

Using the above we now have the following result.

Lemma 3.4 Suppose that Fu is sm in $L$ but not an ideal of $L$. Then either

(i) L is almost abelian; or

(ii) $\langle u, x>$ is a $\mu$-algebra for every $x \in L \backslash F u$.

(iii) $F$ has characteristic two, $L=K$ and $F u=F c$

Proof: Pick any $x \in L \backslash F u$. Then $F u$ is maximal in $\langle u, x\rangle$, by Lemma 2.1. Now let $M$ be a maximal subalgebra of $\langle u, x\rangle$. If $u \in M$ then $M=F u$. So suppose that $u \notin M$. Then $F u$ is a maximal subalgebra of $<u, x>=<u, M>$, whence $F u \cap M=0$ is maximal in $M$, since $F u$ is $\mathrm{lm}$. It follows that every maximal subalgebra of $\langle u, x\rangle$ is one dimensional. The claimed result now follows from Lemma 3.3.

We shall need the following result concerning 'one-and-a-half generation' of rank one simple Lie algebras over infinite fields of characteristic $\neq 2,3$. 
Theorem 3.5 Let $L$ be a rank one simple Lie algebra over an infinite field $F$ of characteristic $\neq 2,3$ and let $F x$ be a Cartan subalgebra of $L$. Then there is an element $y \in L$ such that $\langle x, y\rangle=L$.

Proof: Since $L$ is rank one simple it is central simple. Let $\Omega$ be the algebraic closure of $F$ and put $L_{\Omega}=L \otimes_{F} \Omega$, and so on. Then $L_{\Omega}$ is simple and $\Omega x$ is a Cartan subalgebra of $L_{\Omega}$. Let

$$
L_{\Omega}=\Omega x \oplus \sum_{\alpha \in \Phi}\left(L_{\Omega}\right)_{\alpha}
$$

be the decomposition of $L_{\Omega}$ into its root spaces relative to $\Omega x$. Then, with the given restrictions on the characteristic of the field, every root space $\left(L_{\Omega}\right)_{\alpha}$ is one dimensional (see [2]).

Let $M$ be a maximal subalgebra of $L$ containing $x$. Then $M_{\Omega}$ is a subalgebra of $L_{\Omega}$ and $\Omega x \subseteq M_{\Omega}$. So, $M_{\Omega}$ decomposes into root spaces relative to $\Omega x$,

$$
M_{\Omega}=\Omega x \oplus \sum_{\alpha \in \Delta}\left(M_{\Omega}\right)_{\alpha} .
$$

We have that $\Delta \subseteq \Phi$ and $\left(M_{\Omega}\right)_{\alpha} \subseteq\left(L_{\Omega}\right)_{\alpha}$ for all $\alpha \in \Delta$. As $\left(L_{\Omega}\right)_{\alpha}$ is one dimensional for every $\alpha \in \Phi$, we have $\left(M_{\Omega}\right)_{\alpha}=\left(L_{\Omega}\right)_{\alpha}$ for every $\alpha \in \Delta$. Hence there are only finitely many maximal subalgebras of $L$ containing $x$ : $M_{1}, \ldots, M_{r}$ say. Since $F$ is infinite, $\cup_{i=1}^{r} M_{i} \neq L$, so there is an element $y \in L$ such that $y \notin M_{i}$ for all $1 \leq i \leq r$. But now $\left.<x, y\right\rangle=L$, as claimed.

If $U$ is a subalgebra of $L$, then the normaliser of $U$ in $L$ is the set

$$
N_{L}(U)=\{x \in L:[x, U] \subseteq U\} .
$$

We can now give the following characterisation of one-dimensional semimodular subalgebras of Lie algebras over fields of characteristic $\neq 2,3$.

Theorem 3.6 Let $L$ be a Lie algebra over a field $F$, of characteristic $\neq 2,3$ if $F$ is infinite. Then $F u$ is sm in $L$ if and only if one of the following holds:

(i) Fu is an ideal of $L$;

(ii) $L$ is almost abelian and ad $u$ acts as a non-zero scalar on $L^{2}$;

(iii) $L$ is a $\mu$-algebra. 
Proof: It is easy to check that (i), (ii), or (iii) hold then $F u$ is sm in $L$. So suppose that $F u$ is sm in $L$, but that (i), (ii) do not hold. First we claim that $L$ is simple.

Suppose not, and let $A$ be a minimal ideal of $L$. If $u \in A$, choose any $b \in$ $L \backslash A$. Then $\langle u, b\rangle \cap A$ is an ideal of $\langle u, b\rangle$. Since $0 \neq u \in\langle u, b\rangle \cap A$ and $b \notin A,\langle u, b\rangle$ cannot be a $\mu$-algebra. But then $L$ is almost abelian, by Lemma 3.4, a contradiction. So $u \notin A$. By Lemma 3.3 of [12], $u a=\lambda a$ for all $a \in A$ and some $\lambda \in F$. But now $F u+F a$ is a two-dimensional subalgebra of $\langle u, a\rangle$, a $\mu$-algebra, which is impossible. Hence $L$ is simple.

Now $F u$ is um in $L$ and not an ideal of $L$, so $N_{L}(F u)=F u$, by Lemma 1.5 of [12]. Hence $F u$ is a Cartan subalgebra of $L$, and $L$ is rank one simple. Now $F$ cannot be finite, since there are no $\mu$-algebras over finite fields, by Corollary 3.2 of [6]. Hence $F$ is infinite. But then there is an element $y \in L$ such that $\langle u, y\rangle=L$, by Theorem 3.5 , and $L$ is a $\mu$-algebra. The result is established.

As a corollary to this we have a result of Varea, namely Corollary 2.3 of [14].

Corollary 3.7 (Varea) Let $L$ be a Lie algebra over a perfect field $F$, of characteristic $\neq 2,3$ if $F$ is infinite. If $F u$ is modular in $L$ but not an ideal of $L$ then $L$ is either almost abelian or three-dimensional non-split simple.

Proof: This follows from Theorem 3.6 and the fact that with the stated restrictions on $F$ the only $\mu$-algebras are three-dimensional non-split simple (Proposition 1 of [7]).

\section{Semi-modular subalgebras of higher dimension}

First we consider two-dimensional semi-modular subalgebras. We have the following analogue of Theorem 1.6 of [15].

Theorem 4.1 Let $L$ be a Lie algebra over a perfect field $F$ of characteristic different from 2, 3, and let $U$ be a two-dimensional core-free sm subalgebra of $L$. Then $L \cong s l_{2}(F)$.

Proof: If $U$ is modular then the result follows from Theorem 1.6 of [15], so we can assume that $U$ is not a quasi-ideal of $L$. Thus, there is an element $x \in L$ such that $\langle U, x\rangle \neq U+F x$. Put $V=<U, x>$. Then $U_{V}=U$ implies that $<U, x>=U+F x$, a contradiction; if $U_{V}=0$ then $V \cong s l_{2}(F)$ by Lemma 
2.1 and Theorem 1.6 of [15], and $\langle U, x\rangle=U+F x$, a contradiction. It follows that $\operatorname{dim}\left(U_{V}\right)=1$. Put $U_{V}=F u$. Now $\operatorname{dim}\left(U / U_{V}\right)=1$ and $V / U_{V}$ is three-dimensional non-split simple, by Theorem 3.6 and Proposition 1 of [7]. Thus $V=F u \oplus S$, where $S$ is three-dimensional non-split simple, by Lemma 1.4 of [15], and $F u, S$ are ideals of $V$.

Now we claim that $0 \neq Z(<U, y\rangle) \subseteq U$ for every $y \in L \backslash U$. We have shown this above if $\langle U, y\rangle \neq U+F y$. So suppose that $\langle U, y\rangle=$ $U+F y$. Then $\langle U, y\rangle$ is three dimensional and not simple (since $U$ is two dimensional and abelian), and so solvable. Then, by using Corollary 2.4, we have that $U$ contains a one-dimensional ideal $K$ of $U+F y$ such that $(U+F y) / K$ is two-dimensional non-abelian, and $K=Z(<U, y>)$.

Since $U$ is maximal in $\langle U, x\rangle$ we have $\langle U, x\rangle \neq L$. Pick $y \in$ $L \backslash\langle U, x>$. Then $0 \neq Z(<U, x+y\rangle) \subseteq U$ by the above. Assume that $Z(<u, x>) \neq Z(<U, y>)$. Then $U=Z(<u, x>) \oplus Z(<U, y>)$. Let $0 \neq z \in Z(<U, x+y>)$ and write $z=z_{1}+z_{2}$ where $z_{1} \in Z(<U, x>)$, $z_{2} \in Z(<U, y>)$. Then $0=[z,(x+y)]=\left[z_{2}, x\right]+\left[z_{1}, y\right]$, so $\left[z_{2}, x\right]=$ $-\left[z_{1}, y\right]$. Now, if $z_{1}=0$, then $\left[z_{2}, x\right]=0$, whence $z_{2} \in Z(<u, x>) \cap$ $Z(<U, y>)$, a contradiction. Similarly, if $z_{2}=0$, then $\left[z_{1}, y\right]=0$, whence $\left.z_{2} \in Z(<u, x\rangle\right) \cap Z(<U, y>)$, a contradiction again. Hence $z_{1}, z_{2} \neq 0$. Since $z_{1}, z_{2} \in U$ we deduce that $\left.\left.\left[z_{1}, y\right]=-\left[z_{2}, x\right] \in<u, x\right\rangle \cap<U, y\right\rangle=U$. Thus $y \in N_{L}(U)=U$, a contradiction. It follows that $Z(<U, x>)=$ $Z(<U, y>)$ for all $y \in L$, whence $[L, Z(<U, x>)]=0$ and $Z(<U, x>)$ is an ideal of $L$, contradicting the fact that $U$ is core-free.

Next we establish analogues of two results of Varea from [15].

Theorem 4.2 Let $L$ be a Lie algebra over an algebraically closed field $F$ of characteristic $p>5$. If $U$ is a sm subalgebra of $L$ such that $U / U_{L}$ is solvable and $\operatorname{dim}\left(U / U_{L}\right)>1$, then $U$ is modular in $L$, and hence $L / U_{L}$ is isomorphic to $\operatorname{sl}_{2}(F)$ or to a Zassenhaus algebra.

Proof: Let $L$ be a Lie algebra of minimal dimension having a sm subalgebra $U$ which is not modular in $L$, and such that $U / U_{L}$ is solvable and $\operatorname{dim}\left(U / U_{L}\right)>1$. Then $U_{L}=0$ and $U$ is solvable. Since $U$ is not a quasi-ideal there is an element $x \in L \backslash U$ such that $S=<U, x>\neq U+F x$. Let $K=U_{S}$. If $\operatorname{dim}(U / K)=1$ then $S / K$ is almost abelian, by Theorem 3.6, whence $U$ is a quasi-ideal of $S$, a contradiction. It follows that $\operatorname{dim}(U / K)>1$. If $U / K$ is modular in $S / K$ then $\operatorname{dim}(S / U)=1$, by Theorem 2.4 of [15], a contradiction. The minimality of $L$ then implies that $S=L$. This yields that $U$ is modular in $L$, by Lemma 2.1. This contradiction establishes the result. 
We say that the subalgebra $U$ of $L$ is split if $\operatorname{ad}_{L} x$ is split for all $x \in U$; that is, if $\operatorname{ad}_{L} x$ has a Jordan decomposition into semisimple and nilpotent parts for all $x \in U$.

Theorem 4.3 Let $L$ be a Lie algebra over a perfect field $F$ of characteristic $p$ different from 2. If $U$ is a sm subalgebra of $L$ which is split and which contains the normaliser of each of its non-zero subalgebras, then $U$ is modular, and one of the following holds:

(i) $L$ is almost abelian and $\operatorname{dim}(U)=1$;

(ii) $L \cong \operatorname{sl}_{2}(F)$ and $\operatorname{dim}(U)=2$;

(iii) $L$ is a Zassenhaus algebra and $U$ is its unique subalgebra of codimension one in $L$.

Proof: Let $L$ be a Lie algebra of minimal dimension having a sm subalgebra $U$ which is split and which contains the normaliser of each of its non-zero subalgebras, but which is not modular in $L$. Since $U$ is not a quasi-ideal there is an element $x \in L \backslash U$ such that $S=<U, x>\neq U+F x$. If $S \neq L$ then $U$ is modular in $S$, by the minimality of $L$. It follows from Theorem 2.7 of [15] that $U$ is a quasi-ideal of $S$, a contradiction. Hence $S=L$. Once again we see that $U$ is modular in $L$, by Lemma 2.1. This contradiction establishes the result.

\section{References}

[1] R.K.Amayo, Quasi-ideals of Lie algebras, Proc. London Math. Soc. 33 (1976) 28-36.

[2] G.M.Benkart and J.M.Osborn, Rank one Lie algebras, Ann. of Math. (2) 119 (1984) 437-463.

[3] J.-M.Bois, Generators of simple Lie algebras in arbitrary characteristics, preprint: arXiv:0708.1711 (August, 2007).

[4] K.Bowman and V.R.Varea, Modularity* in Lie algebras, Proc. Edin. Math. Soc. 40 (1997) 99-110.

[5] A.Elduque, On the generators of a semisimple Lie algebra (Spanish), Proceedings of the XIIth Portuguese-Spanish Conference on Mathematics Vol. II (Portuguese)(Braga, 1987) 56-61, Univ. Minho, Braga 1987. 
[6] R.Farnsteiner, On ad-semisimple Lie algebras, J. Algebra 83 (1983) 510519.

[7] A.G.Gein, Modular rule and relative complements in the lattice of subalgebras of a Lie algebra, Sov. Math. 31 no. 3 (1987) 22-32; translated from Izv. Vyssh. Uchebn. Zaved. Mat. 83 (1987), 18-25.

[8] A.A.Lashi, On Lie algebras with modular lattices of subalgebras, J. Algebra 99 (1986), 80-88.

[9] A.Premet, A generalization of Wilson's theorem on Cartan subalgebras of simple Lie algebras, J. Algebra 167 (1994), 641-703.

[10] S.M.Skryabin, Toral rank one simple Lie algebras of low characteristics, J. Algebra 200 (1998), 650-700.

[11] H.Strade, The absolute toral rank of a Lie algebra, Workshop on Lie algebras (Benkart and Osborn, Eds.), Springer Lecture Notes in Mathematics 1373 (1989), 1-28.

[12] D.A.Towers, Semi-modular subalgebras of a Lie algebra, J. Algebra 103 (1986), 202-207.

[13] V.R.Varea, On the existence of ad-nilpotent elements and simple Lie algebras with a subalgebra of codimension one, Proc. Amer. Math. Soc. 104 (1988), 363-368.

[14] V.R.Varea, The subalgebra lattice of a supersolvable Lie algebra, Workshop on Lie algebras (Benkart and Osborn, Eds.), Springer Lecture Notes in Mathematics 1373 (1989), 81-92.

[15] V.R.Varea, On modular subalgebras in Lie algebras of prime characteristic, Contemporary Mathematics 110 (1990), 289-307.

[16] V.R.Varea, Modular subalgebras, quasi-ideals and inner ideals in Lie algebras of prime characteristic, Comm. Algebra 21 (11) (1993), 41954218.

[17] D.J.Winter, The structure of cyclic Lie algebras, Proc. Amer. Math. Soc. 100 (1987), 213-219. 